\title{
Design, inovação e empreendedorismo nos espaços makers
}

\author{
Design, innovation and entrepreneurship in makerspaces
}

\author{
FRANÇA, Rodrigo Braga; Mestrando em Design; UEMG - Escola de Design \\ rodrigo@notusdesign.com.br \\ DE MIRANDA, Carlos Alberto Silva; Doutor; UEMG - Escola de Design \\ carlos.miranda@uemg.br
}

\section{Resumo}

Descrevemos as bases configurativas do Movimento Maker (MM), suas origens e importância na introdução e popularização de novas tecnologias de fabricação digital para o acesso à práticas de aprendizado, experimentação tecnológica e desenvolvimento de novos produtos físicos e digitais, com potencial para ser um vetor de empreendedorismo e inovação. Abordamos o contexto e modelo do 'Fab Lab' - Laboratório de Fabricação digital desenvolvido pelo MIT - enquanto mecanismo de aplicação prática de um novo modelo de projeto e fabricação compartilhada, e refletimos sobre as relações de interação do profissional de design neste novo cenário. Apresentamos como resultado, apontamentos sobre possíveis papeis para a atuação do profissional designer no âmbito dos espaços makers enquanto agente contribuinte para inovação.

Palavras Chave: design; inovação; movimento maker.

\begin{abstract}
We describe the foundations of the Maker Movement (MM), the origins and its importance in the introduction and popularization of new digital manufacturing technologies for the access to learning practices, technological experimentation and new product developing, as a potential driver for entrepreneurship and innovation. We approach the context and model of the 'Fab Lab' - Fabrication Laboratory developed by MIT - as a mechanism of practical application of a new model of sharing design and fabrication, and we reflect on the interaction of the professional designers in this new scenario. We present as a result, indications on possible roles for the professional designer acting within the makerspaces as agents contributing to innovation.
\end{abstract}

Keywords: design; innovation; maker movement. 


\section{Introdução}

A sociedade contemporânea se caracteriza por qualidades como dinamismo, incerteza, fluidez, mudanças, acesso, abundância de informações e tecnologias. Com essa entrelaçada combinação de fatores, característicos da atual realidade complexa, é possível compreender porque a inovação se firmou como um dos valores principais estimados pela nossa sociedade nos últimos tempos, sendo perseguida como forma para solucionar problemas múltiplos e de alta complexidade. Cardoso (2011, p.25) esclarece que 'complexidade' pode ser entendido como um "sistema composto de muitos elementos, camadas e estruturas, cujas inter-relações condicionam e redefinem continuamente o funcionamento do todo".

Na busca por uma inovação social, que valoriza os agentes humanos da inovação e suas características cognitivas com suas capacidades de gerar conhecimento, vivemos também uma alta valorização da criatividade que, em muitos aspectos, é referida como elemento fundamental para construção de soluções e resolução de problemas (DIAS, 2007). A capacidade visionária, a imaginação, a curiosidade, e até mesmo a intuição passam a ser elementos de alta reputação. Características estas que fazem com que o designer se destaque como evidenciado por Neumeier (2010, p.50), que afirma que os designers trabalham através dos problemas, operando no espaço entre o 'saber' e o 'fazer', desenvolvendo protótipos que surgem através da 'empatia', 'intuição', 'imaginação' e 'idealismo'.

Nessa perspectiva, a relevância do design em uma realidade complexa que demanda por inovação é reforçada por Moraes (2008, p.12) que sustenta que "hoje, com o cenário cada vez mais complexo (fluido e dinâmico), é necessário (como nunca) estimular e alimentar constantemente o mercado pela via da inovação e diferenciação do design."

Paralelamente, novos movimentos culturais surgem agregando aspectos de criatividade, tecnologia e também projetuais, como o denominado Movimento Maker, que busca democratizar o acesso a ferramentas projetuais e produtivas para qualquer pessoa com interesse em desenvolver ideias e soluções (GERSHENFELD, 2005). Estes novos movimentos, com característica de "design difuso", ou seja, realizado por designers não profissionais (MANZINI, 2015), reforçam a necessidade de reflexões sobre a atuação do designer contemporâneo, suas necessidades de aprimoramento e novas oportunidades de atuação.

O conteúdo deste trabalho é embasado no chamado Movimento Maker (MM), suas origens e a importância da introdução e popularização de novas tecnologias de fabricação digital para o acesso às práticas de aprendizado, experimentação tecnológica e produção de novos produtos (físicos e digitais), com potencial para ser um vetor de empreendedorismo e inovação (GERSHENFELD, Op. Cit.). O artigo apresenta os makers como, denominado por Anderson (2012), inventores do século XXI e que fazem parte de uma geração que absorveu características da Revolução Digital ${ }^{1}$, com características de aprendizado rápido, troca de conhecimento, facilidade de interação e arranjo em comunidades (TROXLER, 2010).

Neste momento, onde o espaços makers se apresentam como um meio para a inovação ${ }^{2}$,

\footnotetext{
1 A revolução digital é a transição entre a tecnologia analógica e mecânica para a tecnologia digital. A popularização dos computadores pessoais, a internet e a comunicação digital são marcos dessa chamada revolução. Schoenherr, Steven "The Digital Revolution". (5 May 2004), disponível em https://en.wikipedia.org/wiki/Digital_Revolution

2 Inovação segundo Manual de Oslo: "uma inovação é a implementação de um produto (bem ou serviço) novo ou
} 
são abordados os ambientes makers, mais especificamente o modelo 'Fab Lab', modelo de Laboratório de Fabricação digital desenvolvido pelo MIT e que é hoje uma rede internacional, presente em mais de setenta países ${ }^{3}$. Procura-se apresentar suas origens e demonstrar seu caráter orientado pela experimentação tecnológica e aprendizado colaborativo, mas que tem se ampliado a fim de cumprir um importante papel na sociedade, não somente para a experimentação, mas também para uma aplicação prática e inovadora de solução de problemas sociais (tecnologia social) ou através do empreendedorismos de novos negócios (MANDAVILLI, 2006). Buscamos descrever ainda a interface e a participação do profissional de design, neste cenário.

\section{MOVIMENTO MAKER, MAKERSPACES E DESIGN DIFUSO}

O Movimento Maker possui suas raízes no conceito faça-você-mesmo, mas ganhou atualização a partir da introdução de possibilidades trazidas pelas novas tecnologias digitais. Segundo Gershenfeld (2005) e Anderson (2012), a ideia central do Movimento Maker é uma vigorosa atitude para criar e materializar novas ideias (físicas ou digitais) usando máquinas e ferramentas de fabricação digital, como por exemplo impressoras 3d, seja em casa ou espaços de oficinas e laboratórios compartilhados. Para Tanenbaum et al. (2013), Cultura Maker usa artefatos (físicos ou digitais) para explorar um novo presente e futuro de design/projeto, fabricação e consumo. Anderson (Op. Cit.) e Rifikin (2011), estabelecem esta cultura maker e a fabricação digital como importante parte de uma nova, ou terceira revolução industrial capaz de apresentar novas maneiras de desenvolvimento de produtos e negócios.

Gershenfeld (Op. Cit.) e Neves (2014) acreditam que o Movimento Maker é uma nova etapa da 'Revolução Digital' e está alinhado aos princípios desta Era Digital, tais como: colaboração, compartilhamento, atuação em rede, co-criação além da fabricação por meio de softwares de desenvolvimento e manufaturas digitais. Os chamados makers, são considerados por Anderson (Op. Cit.), como parte de uma geração que possuem características de aprendizado rápido, troca de conhecimento, facilidade de interação e arranjo em comunidades, qualidades estas absorvidas da era digital (TROXLER, 2010).

Nesse sentido, makerspaces ou espaços makers, são locais que fornecem um ambiente propício ao desenvolvimento e a experimentação projetual prática. Esses ambientes, munidos e equipados com softwares de projeto 3D e formas de manufatura tecnológicas se arranjam em redes e possuem variadas denominações como Hackerspaces, Innovation Laboratories, Media Labs, Fab Labs, entre outros, mas todos sob o conceito ampliado da democratização do acesso a ferramentas para invenções e expressões pessoais através do compartilhamento de saberes, de tecnologia e de práticas de aprendizado horizontal do Movimento Maker (SMITH et al., 2013). É um dos reflexos da realidade dinâmica da atualidade que incentiva a criatividade e inovação por meio dos avanços tecnológicos.

Alguns desses espaços surgiram a partir da ideia de interação entre universidade pública e empresas, a qual materializou-se, entre outros aspectos, no conceito de parques tecnológicos, ou mesmo incubadoras (GUIMARÃES, 2011). Alguns, localizados junto a universidades e/ou centros de

significativamente melhorado, ou um processo, ou um novo método de marketing, ou um novo método organizacional nas práticas de negócios, na organização do local de trabalho, ou nas relações externas". Disponível em http://www.finep.gov.br/images/apoio-e-financiamento/manualoslo.pdf

${ }^{3}$ Dados da Fundação Fab Lab, disponíveis em http://www.fabfoundation.org/ 
pesquisa. Estes fornecem um ambiente propício ao desenvolvimento e a experimentação prática dos conceitos apresentados acima.

Neves (2014), considera que "estes espaços abertos a todos e acessíveis (tarifas baixas ou mesmo acesso livre) favorecem a redução de barreiras à inovação e à constituição de um terreno fértil a inovação". O conceito que evidencia a importância de um espaço propenso para o surgimento de novas ideias, também é suportado por Peschl e Fundneider (2012) os quais afirmam que a inovação não pode ser fabricada de forma mecânica, sendo então necessário criar um contexto para que a geração do conhecimento e inovação possa surgir, sendo de grande relevância o espaço e ambiente físico que permitam esse processo.

O Movimento Maker possui então, uma proposta convidativa para qualquer pessoa, independente da área de atuação profissional e demais características sócio-culturais, serem agentes ativos de projeto e produção de novos artefatos, modificando conceitos tradicionais de desenvolvimento, produção e consumo de bens (TROXLER, 2010). Segundo Tanenbaum et al. (2013), na nova proposta maker, a criação de softwares inovadores, novas interações e protótipos físicos de produtos não está mais restrita a pesquisadores e profissionais designers bem financiados.

Essa realidade que incentiva e possibilita uma criação, projeto e até mesmo, produção de ideias por atores diversos, não apenas especialistas profissionais, vai de encontro ao que Manzini (2015) chama de designers difusos, indivíduos "comuns" sem expertises acadêmicas e profissionais de design e projeto, mas que assumem, cada vez mais, práticas criativas em suas vidas e atividades como posicionamento perante a um cenário de constante mudanças. Segundo o autor, essas pessoas exercem a atividade projetual em um sentido mais amplo, uma vez que esta é uma habilidade inerente ao ser humano.

\section{REDE FAB LAB}

A natureza e a visão da rede internacional Fab Lab são discriminadas no site da sua Fundação - Fab Lab Foundation ${ }^{4}$. Fab Lab é um modelo de laboratório de fabricação (Fabrication Laboratory) com foco nas tecnologias digitais desenvolvido pelo Massachusetts Institute of Technology (MIT), dentro do laboratório interdisciplinar denominado Center for Bits and Atoms (CBA) sob direção pelo professor Neil Gershenfeld. Este modelo ganhou evidência e se espalhou por diversos países, sendo criada a sua Fundação, sem fins lucrativos, em 2009, como forma de suportar o crescimento internacional da comunidade.

A fundação apresenta os Fab Labs como uma plataforma técnica de prototipagem para inovação e invenção, que compartilha de processos e ferramentas comuns para uma rede distribuída de pesquisa e invenção. Nas exatas palavras da fundação: “Fab Labs são uma rede global de laboratórios locais, permitindo a invenção ao fornecer acesso a ferramentas para fabricação digital" ${ }^{5}$

A compreensão de uma rede de compartilhamento de conhecimento baseada nos conceitos

\footnotetext{
${ }^{4}$ http://www.fabfoundation.org/

${ }^{5}$ Definição do Fab Lab Charter - carta de definições do modelo Fab Lab.

What is a fab lab? Fab labs are a global network of local labs, enabling invention by providing access to tools for digital fabrication
} 
de abertura (open innovation, open design) é essencial para a integração dos Fab Labs.

A Fundação disponibiliza um inventário de maquinário sugerido para os laboratórios, incluindo modelos e valores estimado para a criação de um novo laboratório. Entre as máquinas recomendadas estão: impressoras 3D, máquinas de corte a laser, fresadoras de alta precisão para fabricação de circuitos elétricos e micro controladores e fresadoras de grandes formatos. Todas controladas numericamente por computador (digitais), além de equipamentos e softwares de programação e CAD para desenvolvimento de projetos. Sendo, esses maquinários, de relevante importância para atender às possíveis necessidades encontradas nesses espaços.

Sob a perspectiva do Movimento Maker, o professor e criador do modelo Fab Lab, Neil Gershenfeld, ressalta que os laboratórios são parte de um movimento maior de "fazedores high$t \mathrm{ch}^{\prime \prime}$ que está democratizando o acesso ao apresentar maneiras modernas de fazer coisas, sendo parte de uma nova revolução industrial. (GERSHENFELD, 2005).

Um importante ponto apresentado pela Fundação são seus programas que visam atuação em três áreas distintas: (a) Programa e serviços educacionais, voltado para a disseminação de conhecimento sobre fabricação digital e programas dentro do contexto acadêmico e educacional. (b) Programa de serviços em nível organizacional, para consultoria e acompanhamento na implantação de novos laboratórios, distribuição de informação e conhecimento dentro da rede e gerenciamento da reunião anual de Fab Labs. (c) Programa de serviços voltados à negócios que visa uma maior interação entre iniciativas desenvolvidas dentro de fab labs e novos empreendimentos e negócios. Todos esses, de forma direta ou indireta, colaboram para o desenvolvimento da inovação e empreendedorismo criativo e tecnológico.

Ao avaliar o conteúdo disponibilizado pela fundação Fab Lab em seu site oficial, é possível perceber uma maior orientação para uso da tecnologia como experimentação pessoal ou em grupo. Uma forma de aprendizado ou uma ferramenta para soluções de problemas específicos de determinados grupos e locais. Termos como 'experimentação' e 'invenção' são encontrados repetidamente ao longo das informações disponibilizadas e há grande destaque às máquinas e ao processo de fabricação digital.

Em última análise das informações disponíveis, pode se perceber que expressões como empreendedorismo e inovação, surgem em menor quantidade, sugerindo serem objetivos paralelos desses laboratórios. Apesar do incentivo à processos de co-criação e compartilhamento de saberes, não há menção à métodos e processos organizados e específicos de áreas de maior foco criativo e cognitivo voltadas à projetos, como o design.

\section{EXPERIMENTAÇÃO X EMPREENDEDORISMO E INVENÇÃO X INOVAÇÃO}

Até aqui foi demonstrado que as origens do Movimento Maker partem de uma proposta vinda do faça-você-mesmo, uma atitude de experimentação com grande ênfase nas inovadoras tecnologias digitais. Espaços makers, assim como os Fab Labs, surgiram mais orientados pela tecnologia e troca de conhecimento do que por processos metodológicos específicos de criação e desenvolvimento de projetos. Esse aspecto 'livre' para o desenvolvimento, permite explorar experimentações de novos conceitos como a fabricação pessoal, customizável e compartilhada, ressaltada por Troxler (2010) e Gershenfeld (2005), mas que ao serem confrontados com objetivos

\footnotetext{
${ }^{6}$ No original, Neil Gershenfeld usa a expressão high-tech do-it-yourselfers.
} 
de desenvolvimento de novos negócios e inovações efetivas parecem carecer de metodologias.

Autores como Javelle e Péché (2012) e Smith et al. (2013) discutem se o Movimento Maker é um 'movimento de hobistas high tech ou uma força econômica?'. ${ }^{7} \mathrm{E}$ entre outras proposições acreditam que, cada vez mais, os makers irão combinar atividades de concepção e design, utilizando as ferramentas digitais como formas mais flexíveis e rápidas de prototipagem e testes em etapas de projetos estruturados.

Neves (2014) e Troxler (Op. Cit.) reforçam que Fab Labs, além de laboratórios de manufatura digital, são ambientes físico de socialização, propensos a inovações pela sua prática de abertura e colaboração horizontal entre seus muitos usuários. Contudo Smith et al. (2013) considera que os espaços maker funcionam sobre uma tensão entre projetos e empreendedorismos individuais e atividades coletivas baseadas em uma comunidade.

Em pesquisa realizada em 2013 pelo 'Fab Lab Amsterdã' com seus usuários, foi constatado que, para $75 \%$ dos makers a motivação para uso daquele espaço era trabalhar em projetos pessoais. Já o objetivo principal dos projetos desenvolvidos no Fab Lab, segundo a pesquisa, é 'apenas experimentar' (53\%), nesse caso, bem alinhado com os valores e intenções do Movimento Maker. Entretanto, uma parcela dos usuários (20\%) responderam que o objetivo é a comercialização dos projetos desenvolvidos, demonstrando assim um viés empreendedor, onde a produção destes produtos tem a intenção de gerar valor não apenas para o produtor/maker, mas também para outras pessoas e consumidores.

A pesquisa realizada pelo Fab Lab Amsterdã apresenta como uma de suas conclusões que a ajuda e troca de conhecimento técnico em relação ao uso das máquinas é uma prática diária, mas que isso parece não se refletir quando se trata de colaboração de processos criativos de ideação e desenvolvimento de ideias.

Uma questão relevante a se mencionar, é que cada espaço maker ou mesmo Fab Lab, acaba criando algumas características próprias a partir da comunidade local que o utiliza e da intenção de seus gerenciadores e parceiros locais. Para Mandavilli (2006) enquanto no Fab Lab do MIT os estudantes podem usá-lo para fazer sofisticados gadgets ${ }^{8}$, outras unidades ao redor do mundo estão utilizando o laboratório como oportunidade de implementar ideias que já possuíam e que até então, não havia como ser produzidas, avançando da experimentação para se tornarem ferramentas sérias para enfrentar problemas das nações em desenvolvimento.

Seja através de projetos voltados para comercialização e empreendedorismo, ou em projetos de tecnologias sociais que visam resolver necessidades reais de determinadas regiões, fica claro o potencial destes espaços de irem muito além da experimentação tecnológica.

Nesse novo cenário é preciso distinguir o conceito de invenção e inovação, conceitos estes,

\footnotetext{
${ }^{7}$ Título do artigo de Samuel Javelle and Jean-Patrick Péché- Makers: Hobbyists or a new economic driving force?

Disponível em Http://makingfutures.plymouthart.ac.uk/media/75800/javelle_s__peche_jp_paper.pdf
}

8 Segundo o dicionário Michaelis, Gadget pode significar: Pequeno objeto ou aparelho mecânico ou eletrônico, geralmente de forma e função simples e de utilidade limitada, apesar de mais incomum ou engenhoso do que os dispositivos ou utensílios tecnológicos mais triviais. Disponível em: http://michaelis.uol.com.br/modernoportugues/busca/portugues-brasileiro/gadget/ 
segundo Tidd, Bessant e Pavitt (2008) , frequentemente confundidos devido ao seu significado mais amplo de inovar, derivado do latim innovare, que significa 'fazer algo novo'. Para a inovação efetiva, fazer apenas algo novo, não é suficiente, é preciso transformar ideias em valor, sendo que esse valor deva ser percebido pelas pessoas (PINHEIRO E ALT, 2011).

Schumpeter (1997) desenvolveu a teoria sobre a qualidade e o nível da inovação, e seu trabalho ainda é bastante referenciado por muitos outros autores, determinando a terminologia comumente aplicada a essas métricas (PHLIPEN \& RICCABONI, 2007; MCLAUGHLIN, BESSANT \& SMART, 2007; DARBY \& ZUCKER, 2003; HILL \& ROTHARMEL, 2003; GARCIA \& CANTALONE, 2002 e DOSI, 1982). Algumas expressões que envolvem o conceito de qualidade e nível da inovação são também descritas por Tironi \& Cruz (2008), tais como inovação radical, incremental, imitação, invenção, disruptive, breakthrough, discontinuity, innovation height, novel, novelty, really new, level of newness, innovativeness.

Além disso, a literatura mostra que o retorno privado de uma inovação pode ser muitas vezes mais elevado do que o retorno médio do mercado financeiro (ASTERBO, 2003). Entretanto, a relação entre risco, incerteza e investimento em novos negócios, produtos e tecnologias é demasiadamente complexa.

Nesse contexto de marcante complexidade tecnológica e social, Thackara (2005) alerta sobre o crescente riscode se colocar uma tecnologia inteligente em um produto inútil, de forma que não atenda a nenhuma necessidade atual ou futura. Este autor reforça que não é suficiente apenas fazer coisas tecnicamente incríveis, é preciso explorar necessidades sociais e culturais e também econômicas presentes nas novas mudanças, para que estas se tornem sustentáveis. Como uma das possíveis soluções para este desenredo, o mesmo autor aponta para o design como forma de balancear tecnologia, cultura e atividade humana. Desse modo, um caminho bastante conveniente para viabilizar este processo de inovação é a aplicação de metodologias de design e abordagens projetuais como, por exemplo o "Design Thinking" (BROWN, 2010).

Segundo Brown (2010) e Saher (2017), Design Thinking é uma abordagem estruturada para a criação e o desenvolvimento de novas ideias que busca estabelecer uma consonância entre recursos técnicos disponíveis e os anseios e necessidades humanas.

\section{OPEN-DESIGN, CULTURA MAKER E O DESIGNER}

Ao falar sobre Cultura Maker, um movimento alicerçado em novos conceitos da era digital é importante ressaltar o movimento open source ${ }^{9}$ um movimento marcante deste contexto. Surgido nos anos noventa, esse proposta de projeto aberto mudou a forma de desenvolvimento de softwares propondo um desenvolvimento colaborativo e horizontal, que exalta a criatividade e multiplicidade de agentes participantes do projeto (NEVES,2014). Aplicado ao design, o Open-design pode ser considerado portanto, um projeto aberto de design, passível de ser alterado e construído por diversos atores através de comunidades em redes.

Para Neils Gershenfield (2005), autor do livro "FAB - The Coming Revolution on your Desktop - From personal Computer to Personal Fabrication" e criador da rede 'FAB LABS'10 (Fabrication

\footnotetext{
${ }^{9}$ Código aberto, ou open source em inglês.

10 Fab Labs são uma rede global de laboratórios locais, presente em mais de 70 países e mais de mil unidades, permitindo a invenção e experimentação ao fornecer acesso a ferramentas para fabricação digital.Fonte:Fab Lab
} 
Laboratory), existem similaridades entre os avanços, acesso e popularização ocorridos no universo dos softwares e o que vêm acontecendo em relação à fabricação digital. Para Gershenfield (Op. Cit.) e Troxler (2010), um modelo mais aberto e colaborativo dá poder ao indivíduo, antes, apenas usuário, que passa então a ser um ativo importante no desenvolvimento de ideias e que, juntos a outros agentes através da co-participação, possuem a possibilidade de irem mais longe que individualmente.

Segundo Bastos (2014), o aumento da expansão do conceito Open Design traz impacto profundos, tanto socioeconômicos, como nas práticas do design. Fomentando a inovação em escala global e de forma contínua por permitir que os consumidores tenham autonomia para criar, modificar e produzir produtos totalmente personalizados.

Nesse aspecto, o design como projeto não tem característica restritiva e proprietária, mas é aberto e difuso ressaltando uma mudança de paradigma na atuação do profissional designer apontada por Thackara (2005), onde o designer especialista passa do Projetar Para para Projetar Com, afirmando ainda que a maioria das soluções envolvem alianças e novas conexões.

Isso se relaciona diretamente com o papel contemporâneo do designer segundo reflexões de Manzini (2015), onde há interação e colaboração entre designers experts - aqueles que possuem conhecimento formal - e projetistas não acadêmicos e profissionais, chamados de designers difusos. Tendo o designer especialista uma característica maior de mediador e menor de finalizador, e como defende o autor, a inovação deve ser facilitada pelos designers, ao invés de ser diretamente realizada por eles (MANZINI, 2004).

Os avanços tecnológicos com a popularização das manufaturas digitais e o movimento maker, com incentivo a projetos autorais e disseminação da cultura faça-você-mesmo, estão expandindo as possibilidades de participação ativa da sociedade em geral na cultura material.

Os novos cenários, dinâmicos, abertos e difusos exigem também novas atuações do profissional de design contemporâneo, que pode atuar como proposto por Cardoso (2011, p.243) que defende que "a maior e mais importante contribuição que o design tem a fazer para equacionar os desafios do nosso mundo complexo é o pensamento sistêmico. Poucas áreas estão habituadas a considerar os problemas de modo tão integrado e comunicante".

Essa visão de uma percepção sistêmica que estimula e caracteriza a atuação do design na contemporaneidade é reforçada por Krucken (2008) que complementa que o principal desafio do designer hoje é desenvolver ou suportar o desenvolvimento de soluções para questões de alta complexidade. É nessa atuação ampliada e sistêmica, que deixa o carácter apenas tecnicista e linear, que Moraes (2008) reforça o papel do designer nos novos cenários mutantes e complexos.

Nesse sentido ressalta-se que em um contexto de crescente complexidade tecnológica e social, onde está inserido também o movimento maker, os desafios atuais se apresentam muito além somente das soluções tecnológicas, como observado por Thackara $(2005$, p.4), "nós não podemos parar com a tecnologia, e não há nenhuma razão pela qual devamos. É útil. Mas precisamos mudar a agenda de inovação de tal forma que as pessoas venham antes da tecnologia".

Para a inovação efetiva, fazer apenas algo novo, não é suficiente, é preciso transformar ideias em valor, sendo que esse valor deva ser percebido pelas pessoas (PINHEIRO \& ALT, 2011).

Foundation, disponível em: http://www.fabfoundation.org/ 
Neste aspecto o designer profissional ou especialista, através de suas habilidades, competências e capacitado por conhecimentos metodológicos se faz fundamental para auxiliar a cultura maker dos "fazedores high-tech"11, guiando o processo que possibilite que experimentações possam se desenvolver para inovações com significado e propósito, condições básicas do design.

\section{CONSIDERAÇÕES FINAIS}

Em um cenário difuso onde todos podem criar, projetar, experimentar e fabricar suas ideias ao toque de um mouse e uma impressora 3D, o designer profissional passa então a ter um papel ainda mais importante: ter uma visão sistêmica sobre sociedade, cultura, produtos e sistemas, de modo a auxiliar na garantia de que novas produções sejam carregadas de significado, valores e inovações para a solução de problemas reais e de alta complexidade.

Em perspectiva do tema estudado, em cenários de conexões e redes, marcados por uma criação coletiva e compartilhada como acontece na cultura maker, os designers possuem também a oportunidade de serem, como aborda Manzini (2004), operadores atuantes por dentro de uma rede, de maneira ativa e proativa, de forma a assumir o papel de provedores do processo de inovação. De maneira ampliada, agindo de maneira transversal e entre diferentes disciplinas, o designer deve se posicionar também como gestor da complexidade (MORAES, 2008).

Dentro da mesma visão que coloca o designer na posição de articular, guiar e impulsionar o processo de inovação, o autor e designer Gelli (2012, p.97) afirma que "nessa posição de catalisadores de ideias, poderemos usar todo nosso potencial criativo como maestros dessa revolução que se aproxima, assumindo um papel ainda mais relevante e estratégico no desenho de nosso futuro comum".

Os espaços makers, incluindo os Fab Labs, são considerados, neste artigo, como ambientes de democratização e acesso a fabricação digital com potencial para serem importantes locais de inovação e empreendedorismo ao agregarem metodologias e ferramentas para desenvolvimento criativo de ideias como o Design Thinking. O caráter aberto colaborativo do Movimento Maker e sua atitude convidativa ao criar, experimentar - 'fazer' - vão de encontro com a abordagem do Design Thinking e a mentalidade intrínseca do design que valoriza equipes multidisciplinares e incentiva a visualização e materialização de ideias desde as etapas iniciais do processo.

A manufatura digital tem se popularizado e representado uma quebra de paradigma para a fabricação de novos artefatos, soluções e negócios. O acesso a máquinas e ferramentas digitais, assim como ao conhecimento e informação sobre seus usos têm democratizado a invenção. Apesar disso, somente a experimentação tecnológica, não é suficiente para o desenvolvimento de soluções efetivas para uma realidade social cada vez mais complexa. Nesse sentido, a mentalidade e metodologias do design estruturadas em abordagens como, por exemplo o Design Thinking, se apresentam como uma via apropriada para contribuir no processo dos makers de forma a auxiliar para que as inovações surgidas nos ambientes de projetos tragam soluções e valores reais para uma maior parcela da sociedade.

\footnotetext{
${ }^{11}$ No original, Neil Gershenfeld (2012) usa a expressão high-tech do-it-yourselfers.
} 


\section{BIBLIOGRAFIA}

DIAS, Maria Regina, A. A criatividade na solução de problemas. 4ạ Congresso internacional de pesquisa em design. Rio de janeiro, 2007.

ANDERSON, C. Makers: The New Industrial Revolution. EUA: Random House, 2012.

ASTERBO, T. The return to independent invention: evidence of unrealistic optimism, risk seeking or skewness loving? The Economic Journal, v. 113, issue 484, p. 226, Jan. 2003.

BASTOS F. V. MODA E FABRICAÇÃO DIGITAL EM UM CONTEXTO FAB LAB: Equipamentos, métodos e processos para o desenvolvimento de produtos. Dissertação (Dissetação em design) Universidade Federal de Pernambuco. Centro de Artes e Comunicação. Recife, 2014.

BROWN, Tim. Change by Design How Design Thinking Transforms Organizations and Inspires Innovation. Ed. HarperBusiness, EUA, 2010.

CARDOSO, R. Design para um mundo complexo. São Paulo: Cosac Naify, 2012.

DARBY, M. R.; ZUCKER, L. G. Grilichesian breakthrough: inventions of methods of inventing and firm entry in nanotechnology. July, 2003 (NBER Working Paper, n. 9.825). Disponível em: <http://www.nber.org/papers/w9825>.

DOSI, G. Technological paradigms and technological trajectories: a suggested interpretation of the determinants and directions of technical change. Research Policy, v. 11, p. 147-162, 1982.

FAB LAB Foudantion. < http://www.fabfoundation.org/ >; acessado em 01 de dezembro de 2017.

GARCIA, R; CANTALONE, R. A critical look at technological innovation typology and innovativeness terminology: a literature review. The Journal of Product Management, v. 19, p. 110-132, 2002.

GELLI, Fred. Design e sustentabilidade. Um novo olhar sobre o design brasileiro. São Paulo:

Senai-SP editora; Associação Objeto Brasil, 2012.

GERSHENFELD, N. Fab: The coming Revolution on Your Desktop. Basic Book. 2005.

GUIMARÃES, S. Empreendedorismo intensivo em conhecimento no Brasil. Caderno CRH, 24 (63), pp. 575-591, 2011.

HILL, C. W. L.; ROTHARMEL, F. T. The performance of incumbent firms in face of radical technological innovation. Academy of Management Review, v. 28, n. 2, p. 257-274, 2003.

JAVELLE, Samuel e PÉCHÉ, Jean-Patrick (2014). Makers: Hobbyists or a new economic driving force? Making Futures Journal Vol 3 ISSN 2042-1664 Disponível em

Http://makingfutures.plymouthart.ac.uk/media/75800/javelle_s_peche_jp_paper.pdf de dezembro de 2017.

KRUCKEN, Lia. Competências para o design na sociedade contemporânea. In: MORAES, D.;

KRUCKEN, L. (org.). Cadernos de Estudos Avançados em Design: transversalidade. Belo Horizonte: Santa Clara, Ed UEMG, 2008.

MANDAVILLI, Apoorva. Make anything, anywhere. Nature. Vol. 442. Reino Unido. 2006.

MANZINI, E. El diseño como herramienta para la sostenabilidad medioambiental y social. In:

MACDONALD, S. (org.) Design issues in Europe today. 32 ESTUDOS AVANÇADOS EM DESIGN, 
Caderno2, 2008 Barcelona: BEDA, 2004.

MANZINI, Ezio. Design, when everybody designs: An Introduction to Design for Social Innovation. Cambridge: MIT Press, 2015. 241p.

MCLAUGHLIN, P.; BESSANT, J.; SMART, P. Developing an organizational culture that facilitates radical innovation in a mature small to medium sized company: emergent findings. The Cranfield School of Management Working Paper Series. Disponível em:

<http://www.cranfield.ac.uk/som/research/working_paper>. Acessado em 15 de agosto de 2007.

MORAES, Dijon de. Design e complexidade. In: MORAES, D.; KRUCKEN, L. (org.). Cadernos de Estudos Avançados em Design: transversalidade. Belo Horizonte: Santa Clara, Ed UEMG, 2008.

NEUMEIER, Marty. A empresa orientada pelo design. Tradução: Felix José Nonenmacher. Porto Alegre. Bookman, 2010.

NEVES, H. Maker innovation. Do open design e fab labs; às estratégias inspiradas no movimento maker. Tese (doutorado em Design e Arquitetura) - FAU, Universidade de São Paulo, USP, São Paulo, 2014

PESCHL, F. Markus; FUNDNEIDER, Thomas. Spaces enabling game changing and sustaining innovations: why spaces matters for knowledge creation and innovation. Journal or Organizational Transformation \&amp; Social Change. 2012.

PHLIPPEN, S.; RICCABONI, M. Radical innovation and network evolution. 2007. Disponível em: <http://papers.ssrn.com/sol3/papers.cfm?abstract_id=985887>.

PINHEIRO T., ALT L., Design Thinking Brasil: empatia, colaboração e experimentação para pessoas, negócios e sociedade. Rio de Janeiro. Elsevier, 2011.

RIFKIN, Jeremy. The Third Industrial Revolution: How Lateral Power is Transforming Energy, the Economy, and the world. Palgrave Macmillan, 2011.

SCHUMPETER, J. Teoria do Desenvolvimento Econômico, uma investigação sobre lucro, capital, crédito, juros e o Ciclo Económico. Editora Nova Cultural, São Paulo. 1997.

SMITH, Adriam. HIESCHER, Sabine. DICKEL, Sacha. SODERBER, Johan, VAN OST, Ellen. Grassroots digital fabrication and makerspaces: recofiguring, relocating and recalibrating innovation? SPRU Science and Technology Policy Resarch. 2013.

TANENBAUM, Joshua G. WILLIAMS, M. Amanda. DESJARDINS, Audrey. TANENBAUM, Karen. Democratizing Technology: Pleasure, Utility and Expressiveness in DIY and Maker Practice. Session: Fabrication CHI 2013: Changing Perspectives, Paris, 2013.

THACKARA, John. In the bubble : designing in a complex world. Cambridge, England. The MIT Press. 2005.

TIDD, Joe; BESSANT, John; PAVITT, Keith. Gestão da Inovação. 3ạ edição. Artmed Editora, 2008.

TIRONI, L.; CRUZ, B. Inovação incremental ou radical: há motivos para diferenciar? abordagem com dados da Pintec.Texto para Discussão, Brasília, IPEA, n.1360, 2008.

TROXLER, P. Common-base peer-production of physical goods: is there room for hybrid innovation ecology? in 3a free culture research conference. Alemanha, Berlin, 2010. 